\title{
LA FORMACIÓN PRÁCTICA EN INSTITUCIONES FORMADORAS DE DOCENTES EN LA ARGENTINA
}

\section{Raúl A. Menghini}

Universidad Nacional del Sur

\begin{abstract}
Resumen
En este artículo se problematiza acerca de la formación práctica de los docentes en la Argentina, a partir del reconocimiento de la tensión clásica entre la teoría y la práctica que atraviesa toda política de formación. Esta tensión reconoce una larga historia y se reactualiza ante nuevos escenarios y el desafío de plantear la renovación de los diseños curriculares para la formación del profesorado. El Consejo Federal de Educación (CFE) ha emitido en los últimos años una serie de regulaciones que intentan aportar luz y orientaciones para lograr un cierto equilibrio entre la formación teórica y la práctica; sin embargo la tensión no cesa y, ciertamente, no se resuelve únicamente desde las instancias de gobierno. El campo de la formación de docentes en la Argentina está compuesto por dos circuitos de formación: las universidades y los institutos superiores, cada uno con sus historias y lógicas formativas, políticas, organizacionales y curriculares. Es por ello que en este artículo se presentan y analizan las principales regulaciones nacionales sobre el tema y luego se toman dos casos para analizar cómo se presenta la formación práctica: los diseños curriculares de la Provincia de Buenos Aires y los de la Universidad Nacional del Sur (Bahía Blanca), destacando cómo intentan resolver la tensión planteada en cada una de las instituciones formadoras.
\end{abstract}




\title{
A FORMAÇÃO PRÁTICA EM INSTITUIÇÕES FORMADORAS DE PROFESSORES NA ARGENTINA
}

\begin{abstract}
Resumo
Este artigo problematiza a formação prática dos professores na Argentina, a partir do reconhecimento da clássica tensão entre teoria e prática, que atravessa toda a política de formação. Esta tensão reconhecida historicamente, se renova diante de novos cenários e o desafio de promover a renovação dos currículos de formação de professores. O Conselho Federal de Educação (CFE) emitiu nos últimos anos uma série de regulamentos que tentam fornecer luz e orientação para atingir certo equilíbrio entre a formação teórica e a prática. Contudo, a tensão não cessa e, certamente, não se resolverá unicamente pelas instâncias de governo. O campo da formação de professores na Argentina é composto por dois circuitos de formação: as Universidades e os Institutos Superiores, cada um com suas histórias e lógicas formativas, políticas organizacionais e curriculares. É por isso que neste artigo se apresenta e se analisa as principais regulamentações nacionais sobre o tema, e, em seguida, toma dois casos para analisar como se apresenta a formação prática: os desenhos curriculares da Província de Buenos Aires e os da Universidade Nacional do Sul (Bahia Blanca), destacando como eles tentam resolver essa tensão em cada uma das instituições de formação.
\end{abstract}

Olh@res, Guarulhos, v. 1, n1, p. 40-63, maio. 2013. 


\section{Introducción}

Una de las tensiones clásicas que atraviesan las políticas de formación de docentes remite al equilibrio entre la formación teórica y la formación práctica, cómo irlas graduando y articulando para que los nuevos docentes encuentren sentido a la teoría a la vez que pueden significar la práctica y analizarlas desde marcos teóricos que les permitan comprender particularidades del oficio.

La Argentina no es una excepción en este aspecto y ciertamente esta tensión se ha resuelto de distintas maneras a lo largo de la historia de la formación de docentes y en las últimas décadas ha vuelto a presentarse como un desafío importante para las políticas y los intentos de reforma curricular. No se puede dejar de considerar que el tema suscita intensos debates - desde aquellos que creen que hay que enfatizar la formación teórica porque la práctica se aprenderá en el lugar de trabajo, hasta los que entienden que hay que partir y centrar gran parte de la formación inicial en la práctica- y aún hoy no está absolutamente resuelto, más allá de las regulaciones que se han emitido desde el Consejo Federal de Educación al respecto.

Por otra parte, hay que tener en cuenta la complejidad del campo de la formación de docentes en la Argentina, lo que provoca que haya al menos dos circuitos de formación -o lo que algunos denominan un sistema binario: las universidades -nacionales y privadas- y las instituciones superiores dependientes de las distintas provincias, cada uno con sus propias lógicas políticas, organizacionales y curriculares. En función de esta realidad, en lo que sigue trataremos de realizar algunas precisiones sobre la práctica docente en tanto dispositivo de la formación inicial y revisar las regulaciones nacionales actuales sobre la formación práctica. Finalmente se presentarán dos casos -uno por cada circuito de formación-, para analizar cómo han resuelto y resuelven la tensión planteada en las instituciones de educación superior de la Provincia de Buenos Aires y en una universidad pública como es la Universidad Nacional del Sur, ubicada en la misma provincia, en la ciudad de Bahía Blanca.

Olh@ res, Guarulhos, v. 1, n1, p. 40-, maio. 2013. 
Algunas reflexiones acerca de la práctica docente en contextos de formación inicial

Quizás habría que comenzar diciendo que la formación de docentes no es una excepción si la comparamos con otras formaciones profesionales que han destacado la formación teórica por sobre la formación práctica: baste con citar el caso de los ingenieros, arquitectos, abogados, contadores públicos, asistentes sociales, enfermeros, entre otras. En este sentido, hay que señalar que la formación teórica ha sido la que permitió destacar el status profesional de una determinada carrera, mientras que los problemas de la práctica fueron relegados por entender que son cuestiones que se aprenden en el ejercicio de la actividad profesional. De esta manera, se instaló una racionalidad técnica que subordinó la práctica a la formación teórica. "Esta racionalidad considera, pues, a la práctica como 'campo de aplicación' de fórmulas y técnicas concebidas como universales y verdaderas, en la medida en que se sustentan en conocimientos rigurosos y sistemáticos de base científica" (Davini, 1995: 103).

En otro trabajo hemos afirmado que

“'las prácticas' históricamente han sido consideradas como asignaturas de segundo orden, meras subsidiarias de 'las teóricas'. El prestigio de la teoría frente a la práctica ha sido indiscutible $\mathrm{y}$, en el mejor de los casos, la práctica ha sido entendida como una instancia de aplicación de los saberes generados en el seno de aquella" (Menghini y Negrin, 2008: 5).

Esta situación ha tratado de ser subsanada en las últimas décadas, otorgando a la formación práctica un lugar más destacado y estableciendo porcentajes mínimos para los diseños curriculares de las distintas carreras que implican la realización de distintas instancias de práctica profesional ${ }^{1}$. En esto ha incidido la demanda del mercado laboral y de los propios graduados universitarios que reclamaban una formación más adecuada a la realidad y las actividades propias de cada profesión. Pero aquí aparece otro problema, que consiste en convertir a la práctica en una experiencia cerrada en sí mis-

\footnotetext{
${ }^{1}$ Esto se puede verificar en las carreras de grado y posgrado que han sido sometidas a procesos de acreditación por parte de la Comisión Nacional de Evaluación y Acreditación Universitaria (CONEAU), en los cuales se han tenido en cuenta determinados estándares, en particular aquellos relativos a la formación práctica y las características de la formación teórica.
}

Olh@ res, Guarulhos, v. 1, n1, p. 40-63, maio. 2013. 
ma, como un reportorio del "hacer" profesional, sin posibilidad de analizarla, reflexionarla, articularla con marcos teóricos.

En las últimas décadas las prácticas durante la formación inicial han pasado a considerarse como un eje articulador/vertebrador/integrador que da sentido a toda la formación, y esto se ha visto reflejado en las regulaciones nacionales, así como en las reformas de diseños curriculares para la formación de docentes que fueron gestados por las distintas provincias. No se puede dejar de reconocer que en estas definiciones han influido tanto los aportes de autores relevantes en el ámbito internacional como los de otros nacionales, entre ellos Susana Barco que ya en los '90 planteaba estructurar la formación inicial en dos ejes: uno relativo a la práctica docente y otro sobre el sistema educativo. "Los ejes son pensados en función de los campos disciplinares que la carrera abarca, tanto como de las prácticas profesionales a desarrollar por el egresado y las características del estudiante que cursará la carrera (Barco et al, 2005)" (Barco, 2008: 22). Y en el mismo texto agrega: "el eje se constituye en una matriz generativa distribucional al interior del plan, constituido por conceptos clave que vertebran los elementos nodales de cada campo disciplinar, junto con las prácticas que le son propias" (2008: 22).

En general los diseños curriculares de formación de docentes de las provincias fueron incluyendo espacios de práctica en cada año de formación -siguiendo la tendencia internacional al adelantamiento de éstas-, bajo distintos formatos o dispositivos, en instancias formales e informales, lo que ha permitido una inserción progresiva en el contexto de las organizaciones escolares, para concluir con la "residencia” pedagógica en el último año de formación. “Asistimos, entonces, a una revalorización del lugar y el valor de las prácticas en la formación de docentes, en sintonía con lo que resulta ser las prácticas efectivas que tienen lugar en los más diferentes escenarios" (Menghini y Negrin, 2011: 14).

Pero ciertamente hay que advertir acerca de los riesgos que se pueden generar y es que la inmersión temprana en las prácticas se termine convirtiendo en un proceso adaptativo para la reproducción de las rutinas naturalizadas en la escuela, haciendo creer que esas son las prácticas que se 
espera de los nuevos docentes. Al respecto, Davini afirma que "existe una tendencia muy fuerte a considerar la 'inmersión temprana en el aula' como un proceso de aprendizaje adaptativo a las 'reglas de juego' de la enseñanza, a sus rutinas y a sus rituales, más que como un proceso alternativo y de transformación" (1995: 113). Esto nos lleva a coincidir con la autora en que "la sola inmersión en la práctica no es suficiente. Parece necesario reconstruir la experiencia con marcos conceptuales que orienten la acción" (1995: 113).

En esta temática relativa a la formación práctica de los docentes hay que mencionar también los aportes de Sanjurjo, que realiza una revisión de las distintas tendencias, perspectivas, teorías y tradiciones que se han dado al respecto. La autora rescata los aportes de Schön, Zeichner, Pérez Gómez, Freire, entre otros, y se inscribe en una concepción que rescata fuertemente la reflexión sobre las prácticas desde las tradiciones hermenéutica y crítica. Para ella,

“tomar como eje de la formación docente la reflexión y los procesos metacognitivos se deriva de reconocer la necesidad de que el profesor se desempeñe como un profesional crítico y reflexivo. Por ello explicitamos nuestra concepción acerca de la docencia como práctica profesional" (2002: 39).

En este sentido, las prácticas durante la formación inicial deberían ser un espacio para la reflexión, el análisis, la investigación, a la vez que fuente de interrogación ${ }^{2}$ acerca de la realidad escolar y posibilidad de articulación con los marcos conceptuales. Según Edelstein y Coria, "se impone confrontar permanentemente, a cada paso y no solo al final, desde cada espacio de conocimiento, en el aula o fuera de ella, las construcciones teóricas con situaciones de la práctica concreta" (1995: 22), siempre cuidando de no forzar la práctica y querer atraparla en marcos teóricos que terminen obturando la reflexión que se pretende. En la misma línea, Souto afirma que

"analizar la práctica es referirse a ella, es hacerse la pregunta amplia sobre sus características y sus resultados específicos, sobre sus sentidos para los actores; esa producción ¿en qué cambió la realidad?; ¿qué cosas sucedieron?, ¿qué sentidos se construyeron? Indagar sobre estas preguntas ya corresponde al orden del análisis de la práctica" (2011: 35).

\footnotetext{
${ }^{2}$ Para Souto, "la práctica es fuente de conocimiento singular y su profundización permite teorizaciones. El camino es aquí inductivo: va de la realidad a la posibilidad de teorización y de construcción de conocimientos a partir de ella. La práctica nos formula preguntas, plantea zonas de incertidumbre que permiten formular problemas a resolver, requiere de la invención y la creación y no de la aplicación técnica“" (2011: 35).
}

Olh@ res, Guarulhos, v. 1, n1, p. 40-63, maio. 2013. 
Para que sea posible una práctica reflexiva, se requieren pensar y planificar dispositivos que lo favorezcan, tanto referidos a la práctica en sí misma como a otras instancias en las cuelas ella se convierte en objeto de análisis, teniendo en cuenta que "la formación en las prácticas tiene por objetivo ${ }^{3}$ la preparación para la profesión, por ello es de carácter pre-profesional y toma por objeto las prácticas mismas" (Souto, 2011: 33). Al dispositivo que se plantea en el marco de la formación algunos autores lo denominan "practicum" (Schön, 1992; Blanco, 1999); sin embargo, en la Argentina no se ha impuesto esta denominación, sino que prevalecen otras denominaciones como espacios de la práctica, práctica docente o campo de la práctica ${ }^{4}$, algunas de las cuales van a estar presentes en las regulaciones que analizaremos a continuación.

\section{Regulaciones nacionales sobre la formación en la práctica profesional}

Antes de analizar las regulaciones actuales, haremos una breve referencia a aquellas que surgieron en los 90 con motivo de las reformas educativas de la Ley Federal de Educación 24.195/93, en el marco del neoliberalismo más extremo. Luego de sancionada la ley, se elaboraron los Contenidos Básicos Comunes para la educación básica y para la educación polimodal ${ }^{5}$. A partir de allí, el Consejo Federal de Cultura y Educación (CFCyE) emitió una serie de documentos relativos a la formación de docentes para los distintos niveles o tipos de educación. Así, la Resolución Nro. 53/96 aprobó los Contenidos Básicos Comunes para la formación de docentes que se organizaron en tres campos:

- el campo de la formación general pedagógica

- el campo de la formación especializada por niveles y regímenes especiales

\footnotetext{
${ }^{3}$ El resaltado en itálica corresponde al texto original.

${ }^{4} \mathrm{Si}$ bien cada denominación tiene sus fundamentos epistemológicos, las posibles diferencias no son abordadas en este trabajo.

5 Estas denominaciones obedecen al cambio de estructura del sistema educativo que impuso la ley, transformando así la educación primaria en educación general básica (y llevándola de 7 a 9 años) y la educación secundaria en educación polimodal (acortándola de 5 a 3 años de duración).
}

Olh@res, Guarulhos, v. 1, n1, p. 40-, maio. 2013. 
- el campo de la formación de orientación, que comprendía la profundización centrada en ciclos, áreas y-o disciplinas curriculares (Lengua y Literatura, Matemática, etc.).

Sobre la base de lo resuelto por el CFCyE, luego cada provincia tuvo que elaborar los diseños curriculares para la formación de docentes para los distintos niveles. Algo similar pasó con las universidades, que tuvieron que tener en cuenta estos documentos a la hora de presentar nuevos diseños de formación de profesores.

Nos podríamos preguntar por el lugar que ocupó la formación práctica en esta propuesta. En principio, la práctica la encontramos en el campo de la formación de orientación, o sea en relación con las distintas disciplinas. En cada una de los capítulos (Lengua, Matemática, etc.), el bloque 3 está referido a la "práctica de la enseñanza...". Solo a modo de ejemplo, se enuncian los contenidos que estaban previstos para Lengua y Literatura (pág. 107):

- $\quad$ observación, planificación, conducción y evaluación de procesos escolares de comprensión y producción de textos orales y escritos

- $\quad$ organización de propuestas de actividades para taller de escritura o expresión oral de un grado determinado

- $\quad$ elaboración de un proyecto de biblioteca áulica que promueva la formación de lectores

- $\quad$ planificación de secuencias de actividades para animar la lectura del texto literario.

Si se presta atención a las demás disciplinas, todas ellas contemplan la observación, la planificación y la evaluación. Como se desprende del documento, la práctica está solamente referida a la situación áulica, considerada como 'práctica de enseñanza' y no contempla otras prácticas que incluyan aspectos institucionales, contextuales y sociales.

A pesar de esta concepción limitada de práctica, varias provincias contemplaron en sus diseños curriculares una mirada más amplia de la misma y dedicaron a ella espacios distribuidos a lo largo de los años de formación, como fueron, por ejemplo -sin ser los únicos-, los casos de las provincias de Buenos Aires y Río Negro. En esta última, “el Area de Resi-

Olh@ res, Guarulhos, v. 1, n1, p. 40-63, maio. 2013. 
dencia está desde primer año, deja de tener el espacio de la 'práctica residual' para ocupar un espacio desde la 'práctica que se construye'" (Paugest, 2011: 131). Este tipo de experiencias fue recogida en las regulaciones de este nuevo siglo.

Luego de la crisis político-institucional, social y económica de 2001, y a partir de las autoridades electas según la Constitución Nacional, en 2003 asume la presidencia de la Nación el Dr. Néstor Kirchner. Más allá de las críticas formuladas desde los comienzos de su gestión a las políticas neoliberales de los años 90, en el caso de la educación es recién en 2006 que se impulsa la derogación de la Ley Federal de Educación y la propuesta de una nueva Ley de Educación Nacional. Esta crea el Instituto Nacional de Formación Docente (INFD) como organismo generador y articulador de las políticas de formación de docentes a nivel nacional y también prescribe que la formación inicial de los docentes para cualquier nivel tiene que ser de cuatro años de duración.

Es así como el INFD impulsa la emisión de nuevas regulaciones para la formación de docentes. Entre ellas, la Resolución CFE 24/07 aprueba los "Lineamientos Curriculares Nacionales para la formación docente inicial”, los que luego deberán ser considerados por las distintas provincias para elaborar los nuevos diseños curriculares. Allí se define a la docencia como práctica de mediación cultural, reflexiva y crítica; como trabajo profesional institucionalizado; como práctica pedagógica; y como práctica centrada en la enseñanza. $\left(\# 25^{6}\right)$.

El documento implica un cierto cambio y, en este sentido, "hay que reconocer que, en este caso, se formulan lineamientos curriculares, mientras que en los 90 sólo se habían formulado contenidos básicos" (Menghini, Morales y Aiello, 2009: 432). Si bien ambos documentos prescriben acerca de cómo elaborar los diseños curriculares provinciales (y ambos dan cuenta de políticas centralizadoras), los contenidos de los ' 90 dejaron cierto margen de acción para ello, mientras que estos nuevos Lineamientos acotan mucho

\footnotetext{
${ }^{6}$ Este documento está numerado en párrafos, por eso se seguirá esta forma de citarlo.
}

Olh@res, Guarulhos, v. 1, n1, p. 40-, maio. 2013. 
más las posibilidades de elaborar los diseños, tal como se verá a continuación.

Al igual que en las normativas de los '90, se organiza la formación en tres campos, con la diferencia de que aquí hay un campo dedicado a la práctica docente en particular, a la vez que establecen porcentajes sugeridos para cada uno de ellos:

- campo de la formación general (formación humanística y dominio de marcos conceptuales, interpretativos y valorativos para el análisis e interpretación de la cultura...). De 25 a 35\%.

- campo de la formación específica (disciplinas y didácticas específicas). De 50 a $60 \%$.

- campo de la formación en la práctica profesional, “orientada al aprendizaje de las capacidades para la actuación docente en las instituciones educativas y en las aulas, a través de la participación e incorporación progresiva en distintos contextos socio-educativos" (\#30.3). De 15 a 25\%.

Como se desprende de párrafo citado, prevé que la práctica se desarrolle no sólo en el aula, sino también en otros contextos, y que comience desde el primer año de estudios. Las siguientes afirmaciones son ilustrativas de esta perspectiva:

"Se recomienda que la Formación en la Práctica Profesional acompañe y articule las contribuciones de los otros dos campos desde el comienzo de la formación, aumentando progresivamente su presencia, hasta culminar en las Residencias Pedagógicas" (\#32).

“....apunta a la construcción y desarrollo de capacidades para y en la acción práctica profesional en las aulas y en las escuelas, en las distintas actividades docentes en situaciones didácticamente prefiguradas y en contextos sociales diversos" (\#52).

"Se inicia desde el comienzo de la formación, en actividades de campo (de observación, participación y cooperación en las escuelas y la comunidad), incluyendo la sistematización y análisis de las informaciones relevadas, así como en situaciones didácticas prefiguradas en el aula del Instituto (estudio de casos, análisis de experiencias, microclases)..." (\#53).

En estas citas se puede observar que se pretende una progresión de la práctica en cada año de estudios, de manera de ella abarque experiencias en diversos contextos e instituciones, y que concluya con la Residencia, como un espacio más prolongado de tiempo en el cual se lleva adelante un proceso de intervención pedagógica. De esta manera, el mismo documento señala

Olh@res, Guarulhos, v. 1, n1, p. 40-63, maio. 2013. 
que la práctica debe actuar como "eje integrador" de los diseños curriculares. Por otra parte, se hace referencia a la relación con las escuelas en donde los estudiantes desarrollarán sus prácticas, y propone la generación de Redes entre instituciones formadoras y escuelas, denominando a estas últimas como "escuelas asociadas".

A fin de orientar a las provincias, el INFD difundió el documento "Recomendaciones para la elaboración de Diseños Curriculares. Recomendaciones sobre el campo de la formación en la práctica profesional". En él se afirma que "la práctica profesional es concebida como un conjunto de procesos complejos y multidimensionales asociados a todas aquellas tareas que un docente realiza en su puesto de trabajo" (pág. 2). También hace una recuperación de las experiencias de las provincias que ya habían incluido la práctica desde los inicios de la formación, aunque al mismo tiempo señala las posibles limitaciones, dado que más práctica no garantizó un cambio sustancial para la formación ni para el desempeño profesional. Al respecto, advierte que "el espacio de la formación en la práctica debe proveer a los futuros docentes esquemas conceptuales y prácticos que no se diluyan en el proceso de socialización profesional” (pág. $5)$.

El documento citado culmina con una propuesta, a modo de ejemplo, de cómo las provincias podrían secuenciar la formación práctica:

- primer año: visitas a las escuelas y participación en algunas actividades escolares. Rotación en distintos ámbitos socio-educativos, - segundo año: participación en actividades de responsabilidad creciente, como observación participante en las aulas y colaboración en actividades docentes en aula,

- tercer año: programación y desarrollo de clases específicas en las aulas, con la guía del profesor de práctica y el docente orientador, - cuarto año: residencia pedagógica con rotación por cursos de los distintos ciclos del nivel educativo (inicial, primario, secundario). 
En todos los casos propone que las actividades realizadas en el ámbito escolar sean acompañadas con espacios para el análisis y la reflexión bajo la forma de seminarios, talleres, ateneos. Allí se trabajaría con la información recogida en los ámbitos concretos de las prácticas docentes, para lo cual se sugiere utilizar la observación, entrevistas, análisis documental, búsqueda bibliográfica, entre otras.

De acuerdo a lo que se planteó en la introducción de este trabajo, analizadas las regulaciones nacionales actuales para elaborar los diseños curriculares y considerar en ellos la formación práctica, a continuación se presentará cómo viene resolviendo este tema la provincia de Buenos Aires y qué sucede en el caso de la Universidad Nacional del Sur (Bahía Blanca).

\section{La formación práctica en los diseños curriculares de la Provincia de} Buenos Aires

La provincia de Buenos Aires incluyó la formación práctica en cada año de formación ya hacia fines de los '90, antes de que la regulación nacional actual lo prescribiera (Resoluciones 4508/97, la 3100/98 y la 13271/99 de la Dirección General de Cultura y Educación). En esos diseños curriculares se destaca el espacio dedicado a la práctica docente desde primer año en tanto “eje vertebrador" de la formación. Sin embargo, se puede observar una gran contradicción entre los marcos teóricos sobre los que se basa -en general en la línea de una pedagogía crítica- y el enfoque sobre la práctica docente que presenta una impronta aplicacionista de la teoría y naturalizadora de la realidad, toda vez que no apunta a revisarla críticamente sino que hace de los docentes meros solucionadores de problemas desde el punto de vista técnico, con vistas al mejoramiento pero no a la transformación de la realidad. En otro trabajo hemos afirmado que "no basta con pensar en introducir más práctica, sino que se hace necesario repensar qué tipos de prácticas, con qué sentido, en qué articulación con la teoría, en relación con qué estrategias y formas de reflexión” (Menghini y Morales, 2007: 237). Un análisis similar realiza Insaurralde, cuando afirma y se pregunta: 


\begin{abstract}
"la transformación educativa ha exacerbado la práctica de introducir planteos originados en las tradiciones progresistas de la educación. Un ejemplo de ello es la extensión del término 'praxis', 'reflexión sobre la práctica' o 'práctica investigativa del docente'. ¿Es que la 'transformación de la formación docente" se compromete con las propuestas de Schön, Stenhouse o de las perspectivas críticas? ¿Qué sentido cobran aquellos planteos en la reforma del currículo de formación de docentes? ¿Se han incluido los conceptos junto a su significado original? O por el contrario, ¿aquellas conceptualizaciones han sido vaciadas de contenido?" (Insaurralde, 2002:52).
\end{abstract}

Entre los años 2006 y 2007 la provincia de Buenos Aires inicia la revisión de sus diseños curriculares (Res. 3.655/07) para adecuarlos a la nueva normativa nacional que se analizó en el apartado anterior, comenzando con la formación para nivel inicial y primario. Luego fueron cambiando también la formación de profesores especiales y de educación física, restando otros profesorados para nivel secundario. En los nuevos diseños la formación se organiza en cinco campos $^{7}$, siendo uno de ellos el de la práctica -antes denominado espacio de la práctica-, que es considerado articulador y está compuesto por tres componentes: las herramientas de la práctica, la práctica en terreno y el taller integrador e interdisciplinario (TAIN) ${ }^{8}$.

Si bien todavía faltan algunos diseños para la formación para nivel secundario, seguramente seguirán la misma lógica que los de nivel primario, de allí que se tomará en cuenta este nivel para ilustrar cómo se organiza este campo en cada año de formación.

- Primer año: indica que en la práctica en terreno,

"el futuro docente se vincule con el campo sociocultural de la comunidad, a través de las organizaciones de la misma, en una práctica educativa no escolarizada y no reducida a acciones de apoyo escolar. Tiene por objeto acceder a la práctica docente desde la comprensión y el posicionamiento del futuro docente en el campo educativo" (DGCyE, 2007:58).

En las herramientas de la práctica se debe trabajar un "Taller de educación social y estrategias de educación popular” y en el TAIN se aborda el concepto de "Ciudad Educadora".

\footnotetext{
${ }^{7}$ Coincidimos con Insaurralde y Agüero en que "el término Campo de formación no es definido en los documentos de las reformas de formación de docentes y no reconoce antecedentes en las teorías curriculares" (2009: 222)

${ }^{8}$ Este taller se presenta como un espacio para la reflexión y construcción compartida entre todos los actores implicados: alumnos, docentes de los diferentes campos, integrantes de las instituciones contactadas para la práctica en terreno.
}

Olh@res, Guarulhos, v. 1, n1, p. 40-, maio. 2013. 
- Segundo año: la práctica en terreno se desarrolla en las instituciones de cada nivel, atendiendo a diferentes realidades: urbana, suburbana y rural. La intención está puesta en una aproximación a las instituciones y sus contextos, desde una perspectiva cualitativa. Es por ello que el eje de las herramientas de la práctica se denomina "Aproximación y análisis cualitativo institucional" y el TAIN se centra en el espacio escolar y la realidad educativa.

- Tercer año: se presenta el abordaje de la problemática educativa en el aula. Se deben atender también a los diferentes contextos y modalidades incluidas en la Ley Provincial de Educación. Las herramientas de la práctica abordan los contenidos en referencia a "la investigación en y para la acción docente", y el eje del TAIN es la relación educativa.

- Cuarto año: prevé la práctica específica en el aula que toma la forma de residencia. El TAIN, por su parte, hace hincapié en el "Posicionamiento docente" y aquí no hay previstas herramientas de la práctica específicas.

En un trabajo anterior (Menghini, Díaz, Gardié, Iriarte, 2011) señalábamos dos problemas de difícil resolución a la hora de implementar estos diseños:

1. la posibilidad de realizar prácticas en terreno en distintas realidades e instituciones, básicamente por la falta de recursos económicos que hagan posible desplazamientos en la ciudad y en zonas rurales.

2. la implementación del TAIN que, si bien se intenta garantizar con una asignación semanal plus ${ }^{9}$, resulta muy complejo hacerlo posible y las instituciones formadoras han tenido que buscar las más diversas alternativas. Aquí podemos coincidir con otras autoras que han observado problemas similares en distintas instituciones de la Provincia. Así, Oreja Cerruti afirma que "en las actuales condiciones de trabajo de sobrecarga de tareas y superposición de instituciones por parte de los docentes, se hace dificultosa la concreción sistemática de

\footnotetext{
${ }^{9}$ Los docentes formadores reciben el pago de horas plus en función de la carga horaria que tengan, y esas horas deben ser utilizadas para la participación en este Taller.
}

Olh@res, Guarulhos, v. 1, n1, p. 40-63, maio. 2013. 
dicho taller" (2009:246-247)". E Insaurralde cuestiona que sea posible desarrollar un taller con un gran número de docentes y estudiantes, además de que "no todos pueden concurrir el mismo día y en la misma franja horaria" (2011: 118). Respecto de este espacio, hemos aventurado que los problemas de concreción

"pueden llevar a que en el futuro este Taller desaparezca si no logra los objetivos que pretende, y es muy posible que la culpa recaiga -como tantas veces ha sucedido- en los propios docentes formadores y terminen siendo acusados de incapaces de defender este tipo de espacios" (Menghini, Díaz, Gardié, Iriarte, 2011).

\section{La formación práctica de los docentes en la Universidad Nacional del}

\section{Sur}

Como hemos sostenido más arriba, la formación de profesores para nivel secundario en las universidades no ha seguido el ritmo de las transformaciones que se impulsaron desde la Nación durante los '90 y en la primera década del siglo XXI. Sea porque en general son instituciones en cierto sentido más "conservadoras" y tienen una particular forma de entender el avance del conocimiento científico y su proyección en la formación, o bien porque en aras de su autonomía intentan mantenerse al margen de las orientaciones nacionales y hacer sus propios caminos, lo concreto es que en general las universidades no cambian sus planes de estudio con la periodicidad que hoy sería deseable ${ }^{10}$.

Mollis (2009) señala que

existen dos tipos de "tradiciones académicas" desde el punto de vista de la formación de profesores universitarios: las tradiciones académicas que ponen el énfasis en la formación disciplinar y las que ponen énfasis en la formación pedagógica. Ambas tradiciones son reconocidas como visiones o ideologías que dominan tanto la organización y distribución de las materias del plan de estudios como las percepciones que tienen los profesores sobre sus prácticas y las expectativas de formación de los futuros egresados.

\footnotetext{
${ }^{10}$ Hay que tener en cuenta que, siendo autónomas, las universidades tienen su propio gobierno y la vida democrática no permite cambios tan rápidos. En contraposición, las instituciones superiores provinciales dependen de los ministerios - en tanto áreas del poder ejecutivo-, y tienen la posibilidad de generar cambios centralizados pasando por alto muchas veces la lógica de la vida democrática institucional.
}

Olh@res, Guarulhos, v. 1, n1, p. 40-, maio. 2013. 
Sin embargo, la tensión entre estas dos tradiciones en las universidades parece ceder a favor de la académica. La investigación de campo realizada por la autora ${ }^{11}$ recién citada le permite afirmar que "hubo un reconocimiento generalizado de la falta de valoración institucional hacia los espacios para la práctica de la enseñanza. En las diferentes tradiciones de trayectos formativos para los profesores, las prácticas de la enseñanza constituyen el punto débil”.

Por otra parte, en la mayoría de las universidades el eje pasa por las disciplinas y, a partir de ellas, se ofrece formación para profesorados habilitados formalmente para la enseñanza- y para licenciados -habilitados para la investigación científica-, compartiendo ambas formaciones una parte importante de los respectivos planes de estudio. Como afirman Pogré et $a l^{12}$, "tradicionalmente las propuestas curriculares de formación se caracterizaron por constituir un agregado de asignaturas que, por lo general, referían cada una de ellas a un campo disciplinario. Esta tradición se ha conservado -a pesar de los procesos de reforma de los últimos años-, mientras que en otros empieza a mostrar variaciones" (2005: 32-33).

Asimismo, la investigación de estas autoras les permitió observar que los profesorados de Matemática y Física en general contaban con más cantidad de materias pedagógicas (21 a 30\%) que los de Historia (de 11 a 20\%), y que en estas materias estaban incluidas las instancias de práctica docente. También pudieron concluir que todavía un gran número de planes de estudio ubica las asignaturas pedagógicas y la formación práctica en el último año de formación, con lo cual "sigue vigente el modelo deductivo que asume que las instancias de práctica constituyen un espacio de 'aplicación' de lo aprendido" (Pogré, 2005: 39).

En el caso de la Universidad Nacional del Sur, se verifican gran parte de las conclusiones de las autoras. Si se revisa la historia de los diseños curriculares del profesorado en Letras, por ejemplo, encontramos que el

\footnotetext{
${ }^{11} \mathrm{Su}$ investigación se basa en la selección de un instituto superior de la provincia de Córdoba y cuatro universidades: tres nacionales (Buenos Aires, Córdoba y Patagonia Austral) y una privada (Salvador).

${ }^{12}$ Las autoras analizaron los diseños curriculares de profesorados en Matemática (en 23 universidades), Historia (en 17 universidades) y Física (en 14 universidades).
}

Olh@ res, Guarulhos, v. 1, n1, p. 40-63, maio. 2013. 
primer plan data de $1956^{13}$ y estuvo vigente hasta 1967, para luego cambiar en el marco de la Revolución Argentina (gobierno de facto del Gral. Onganía). Otro cambio se va a producir en 1976, meses previos al golpe de estado que encabezó el Gral. Videla. En ambos casos, los estudiantes primero obtenían el título de licenciado y luego podían cursar un año más Pedagogía General, Didáctica fundamental y especial, Psicología evolutivay la práctica docente, denominada en ese momento "práctica de la enseñanza" en la disciplina- y obtener el título de profesor.

En 1981, todavía en el marco de la última dictadura, se cambia nuevamente el plan de estudios distinguiendo ambos títulos (licenciatura y profesorado), pero conformando un ciclo común los primeros cuatro años y diferenciando la formación en el último: materias pedagógicas para los profesorados; seminarios de intensificación teórica para los licenciados. En cualquiera de los cambios señalados en las distintas décadas se pueden vislumbrar más continuidades que rupturas:

el énfasis está puesto en el dominio de las bases teóricas de la disciplina, lo que estaría dando legitimidad a la formación, mientras que lo pedagógico resulta secundario, a manera de instrumentos o herramientas para llevar adelante los conocimientos científicos en la escuela secundaria. (Menghini, Vuela e Irazabal, 2009).

Por fin, en 2001, se realiza el último cambio curricular que obedeció a varias causas: la necesidad de dejar atrás los planes formulados durante la última dictadura, una actualización de los conocimientos, los cambios generados en el sistema educativo y las normativas derivadas de las Leyes educativas del gobierno del Dr. Menem.

Es posible advertir que en estos planes aparece con claridad una mayor injerencia del Ministerio de Educación, ya que para su elaboración -en el Departamento de Humanidades- y para su aprobación -por parte del Ministerio- se consideraron las resoluciones del Consejo Federal de Cultura y Educación. Por otra parte, los planes contemplan algunas materias que se adecuan a las modificaciones que sufrió la educación media en la década, en particular en la Provincia de Buenos Aires. (Menghini, Vuela e Irazabal, 2009).

\footnotetext{
${ }^{13}$ Hay que recordar que la misma Universidad Nacional del Sur es creada en 1956 sobre la base del entonces Instituto Tecnológico del Sur. En ese momento, asume los planes de estudio que estaban vigentes en dicho Instituto.
}

Olh@res, Guarulhos, v. 1, n1, p. 40-, maio. 2013. 
En los nuevos planes se destaca el incremento de la formación pedagógica, que pasó de tres materias a siete: Teoría Educativa, Didáctica General, Psicología Educacional, Psicología Evolutiva, Didáctica de la Lengua y la Literatura, Seminario de Perspectivas pedagógicas de la educación superior, Seminario de política y legislación de la educación superior; y se agregó la práctica en el nivel superior de la enseñanza, con lo cual actualmente existen dos espacios de práctica: uno anual para el nivel secundario y otro cuatrimestral para el nivel superior, ambos en el último año de formación. Estas modificaciones obedecieron, en gran parte, a las regulaciones nacionales de la década del 90 -que continuaron vigentes hasta aproximadamente 2005- y que se inscribieron en el marco de las Leyes Federal de Educación y de Educación Superior, las dos del período más fuertemente neoliberal.

A pesar de las nuevas materias pedagógicas y su inclusión en los distintos años de la formación de profesores, hay que advertir que los espacios de práctica siguen estando en el último año de formación, manteniéndose fuertemente el sentido aplicacionista de la teoría en la práctica.

En el caso de la práctica en el nivel secundario, su carácter anual permite que los estudiantes se inserten en las instituciones educativas públicas y permanezcan allí durante varios meses (aproximadamente de abril a octubre), realizando actividades diversas para lograr un conocimiento del contexto, la institución y las características del grupo áulico. En general se conforman grupos de tres o cuatro estudiantes de distintas disciplinas ${ }^{14}$ que concurren a una escuela que les es asignada.

Todo el período se estructura en tres etapas que suponen distinto tipo de actividades:

- de inserción y conocimiento contextual, institucional y del aula: desde abril a julio

- observaciones intensivas: julio-agosto

- $\quad$ residencia pedagógica: agosto a octubre

\footnotetext{
${ }^{14}$ En la Universidad Nacional del Sur se forman profesores en Letras, Historia, Filosofía, Geografía, Matemática, Química, Economía, Física, Geociencias.
}

Olh@res, Guarulhos, v. 1, n1, p. 40-63, maio. 2013. 
Durante la primera etapa van realizando distintos trabajos prácticos que incluyen una parte grupal y otra individual, básicamente centrada en la realidad del aula en la cual están observando. Deben asistir al menos una vez a la semana a la escuela en forma obligatoria, más todas las veces que resulten necesarias para recabar información, realizar entrevistas, participar en actividades institucionales. La segunda etapa supone la asistencia a la escuela en todos los horarios de clase de la materia en la cual realizará la residencia. Y finalmente, la residencia implica el dictado de entre quince y veinte clases a cargo de los residentes.

A partir de la segunda etapa, los residentes comienzan a elaborar la propuesta didáctica que llevarán adelante y para ello cuentan con el asesoramiento de los profesores de la universidad, en particular de aquellos que dictan las didácticas específicas. Ellos son los responsables de aprobarles el plan general de intervención didáctica y el de cada clase en particular. Luego, durante la realización de la residencia, serán observados por los profesores de práctica y también por los profesores de las didácticas de manera conjunta.

En forma simultánea a la concurrencia a las escuelas, los estudiantes asisten a clase a la universidad donde se va trabajando alrededor de distintas dimensiones de la práctica docente (política, institucional, didáctica, entre otras) y se van realizando ejercicios de análisis de las prácticas docentes con distintos dispositivos y en articulación con marcos teóricos.

En la práctica para el nivel superior, deben realizar una inserción institucional en institutos superiores públicos o privados, y en ellos realizan un período de observación y luego dictan algunas clases. Al igual que la práctica para nivel secundario, los estudiantes asisten a clases en la universidad donde se proponen actividades de análisis de lo que observan en los institutos y de la intervención docente que han realizado.

Si bien se puede percibir que ha habido un crecimiento de los espacios de práctica en la universidad y que la misma tiene otra valoración, todavía falta mucho por lograr, especialmente quitarle el carácter residual y aplicacionista que sigue primando, a la vez que comprometer a los formadores de las

Olh@res, Guarulhos, v. 1, n1, p. 40-, maio. 2013. 
disciplinas en la necesidad de tensionar la formación teórica hacia las instancias de la práctica docente.

\section{Un cierre para continuar pensando}

Hemos observado que frente a las críticas de la debilidad de la formación práctica de los docentes, ha habido un movimiento por parte de docentes e investigadores que han instalado este problema en la agenda pública y ésta ha sido retomada por los organismos del Estado encargados de formular las políticas para la formación de docentes. Resulta sumamente valorable el avance que ha tenido en términos curriculares la práctica docente, como eje articulador, integrador o vertebrador de toda la propuesta curricular, y la posibilidad de que los nuevos aspirantes a docentes se formen con una inserción temprana en la realidad de las escuelas, ya desde el comienzo de los estudios en el caso de las instituciones superiores provinciales formadoras de docentes. Y si bien esto todavía no sucede en el caso de las universidades -entre otras cosas, por su tradición fuertemente academicista-, es posible que en los próximos cambios de diseños curriculares con motivo de los procesos de acreditación de los profesorados universitarios, haya un avance en tal sentido y los espacios de práctica logren ganar terreno.

Sin embargo, también hay que señalar las limitaciones de las regulaciones nacionales o provinciales e incluso de los diseños curriculares, porque éstas de por sí no cambian la realidad de la formación. Además de estas iniciativas, hace falta trabajar hacia adentro de las instituciones formadoras, generar otra conciencia entre los docentes formadores, para poder buscar formas concretas y creativas de tensionar y articular la formación teórica con la formación práctica, porque de lo contrario -y a pesar de los cambiosse seguirán manteniendo como dos campos separados que marchan por caminos separados pero no se cruzan.

En este sentido, conviene asimismo reparar en que la sola inclusión de más espacios de práctica no garantizan que se vaya a superar el sentido aplicacionista de las mismas, y para ello se requiere continuar intentando formatos curriculares, dispositivos concretos de trabajo que apunten a la

Olh@ res, Guarulhos, v. 1, n1, p. 40-63, maio. 2013. 
generación de experiencias de práctica que se vean enriquecidas con aportes teóricos y metodológicos, de manera de hacer real la tan mentada práctica reflexiva, ya desde la formación inicial, para que luego la puedan sostener una vez que los nuevos docentes estén trabajando en las escuelas. Así, será posible pensar en la mejora de la educación, en la medida que se logre formar docentes con sentido de realidad a la vez que sólidos en su formación teórica y con capacidad para enfrentarse críticamente a las problemáticas de la escolarización de niños y adolescentes. 


\section{Bibliografía}

Barco, Susana (2008) "Prácticas y residencias docentes: viejos problemas, ¿nuevos enfoques? Historias con Matrioshkas”. En Menghini, Raúl y Negrin, Marta (comps.) Prácticas y residencias docentes. Viejos problemas, ¿nuevos enfoques? Ediuns. Bahía Blanca.

Blanco, Nieves (1999) “Aprender a ser profesor/a: el papel del practicum en la formación inicial”, en Angulo Rasco, J. F.; Barquín Ruiz, J. y Pérez Gómez, A. I., Desarrollo profesional del docente: política, investigación y práctica, Akal, Madrid.

Davini, María C. (1995) La formación docente en cuestión: política y pedagogía. Paidós. Buenos Aires.

Insaurralde, Mónica (2002) "El proceso de desarrollo curricular del 'Espacio de la práctica docente"”. Tesis de la Maestría en Didáctica, Universidad de Buenos Aires, Facultad de Filosofía y Letras, mimeo.

Insaurralde, Mónica (2011) "Políticas curriculares para la formación de docentes de Educación Primaria en la Provincia de Buenos Aires”. En Menghini, Raúl y Negrin, Marta (comps.) Prácticas y residencias en la formación de docentes. Baudino ediciones. Buenos Aires.

Insaurralde, Mónica y Agüero, Claudia (2009) "La formación de profesoras/es para la Educación Primaria: las decisiones centrales de los gobiernos nacionales de la década de los '90 y los post '90. ¿Cambios o enmascaramientos?’. En Vior, Susana; Misuraca, M. Rosa y Más Rocha, Stella (comps). Formación de docentes. ¿Qué cambió después de los '90 en las políticas, los currículos y las instituciones?, Buenos Aires, Jorge Baudino Ediciones.

Menghini, Raúl; Díaz, Mabel; Gardié, Silvana e Iriarte, Laura (2011) ““"Política curricular de formación de docentes en la Provincia de Buenos Aires". VI Jornadas Nacionales sobre formación del profesorado. Universidad Nacional de Mar del Plata. Mar del Plata. http://www.mdp.edu.ar/humanidades/pedagogia/jornadas/jprof2011/mesas.htm\#5.

Menghini, Raúl y Morales, Laura (2007) "La formación de docentes de la provincia de Buenos Aires: análisis del espacio de la práctica”. En Burgos, Nidia y Rigatuso, Elizabeth (ed.) La modernización del sudoeste bonaerense: reflexiones y polémicas en el ámbito educativo, lingüístico y literario. Universidad Nacional del Sur. Bahía Blanca.

Olh@ res, Guarulhos, v. 1, n1, p. 40-63, maio. 2013. 
Menghini, Raúl; Morales, Laura y Aiello, Berta (2009) "Diseños curriculares para la formación de docentes en la Provincia de Buenos Aires a la luz de las nuevas regulaciones nacionales y provinciales". En Cernadas, Mabel y Marcilese, Juan (editores) Política, sociedad y cultura en el Sudoeste Bonaerense (Actas V Jornadas Interdisciplinaria del Sudoeste Bonaerense) EdiUNS. Bahía Blanca. Pág. 429/439.

Menghini, Raúl y Negrin, Marta (comps.) (2008) Prácticas y residencias docentes. Viejos problemas, ¿nuevos enfoques? Ediuns. Bahía Blanca.

Menghini, Raúl y Negrin, Marta (comps.) (2011) Prácticas y residencias en la formación de docentes. Baudino ediciones. Buenos Aires.

Menghini, Raúl; Vuela, Emiliano e Irazabal, Ana (2009) "Políticas y diseños curriculares para la formación de profesores en la universidad. Posibilidades y obstáculos". II Jornadas de Formación docente universitaria. Facultad de Humanidades y Artes. Universidad Nacional de Rosario. Rosario.

Mollis, Marcela (2009) "La formación de profesores universitarios para el nivel medio y superior: una asignatura pendiente”. La revista del CCC [en línea]. Enero / Agosto 2009, n 5 / 6. Disponible en Internet: www.centrocultural.coop/revista/articulo/109/.

Oreja Cerruti, Betania (2009) "Reforma curricular en la formación de maestros de la provincia de Buenos Aires". En Vior, Susana; Misuraca, M. Rosa y Más Rocha, Stella (comps). Formación de docentes. ¿Qué cambió después de los '90 en las politicas, los currículos y las instituciones?, Buenos Aires, Jorge Baudino Ediciones.

Paugest, Alicia (2011) "La formación docente en la Provincia de Río Negro: una mirada retrospectiva sobre los Diseños Curriculares“. En Menghini, Raúl y Negrin, Marta (comps.) Prácticas y residencias en la formación de docentes. Baudino ediciones. Buenos Aires.

Pogré, Paula et al (2005) Formar docentes. Una alternativa interdisciplinar. Papers editores. Buenos aires.

Sanjurjo, Liliana (2002) La formación práctica de los docentes. Reflexión y acción en el aula. HomoSapiens. Rosario.

Schön, D. (1992) La formación de profesores reflexivos, Paidós, Barcelona. 
Souto, Marta (2011) "La residencia: un espacio múltiple de formación”. En Menghini, Raúl y Negrin, Marta (comps.) Prácticas y residencias en la formación de docentes. Baudino ediciones. Buenos Aires.

\section{Documentos}

Instituto Nacional de Formación Docente. "Recomendaciones para la elaboración de Diseños Curriculares. Recomendaciones sobre el campo de la formación en la práctica profesional”.

Provincia de Buenos Aires. Dirección General de Cultura y Educación. (2007). Resolución Nro. 3.655. “Diseño curricular de formación docente. Niveles inicial y primario”.

República Argentina. Ley Federal de Educación No 24.195/93

República Argentina. Consejo Federal de Cultura y Educación. Resolución N 53/96.

República Argentina. Consejo Federal de Educación. Resolución No 24/07. 